\title{
Signatures of strong correlation effects in resonant inelastic x-ray scattering studies on cuprates
}

\author{
Wan-Ju Li, ${ }^{1, *}$ Cheng-Ju Lin, ${ }^{1,2}$ and Ting-Kuo Lee ${ }^{1, \dagger}$ \\ ${ }^{1}$ Institute of Physics, Academia Sinica, Taipei 11529, Taiwan \\ ${ }^{2}$ Department of Physics and Institute for Quantum Information and Matter, California Institute of Technology, \\ Pasadena, California 91125, USA
}

(Received 18 April 2016; revised manuscript received 21 July 2016; published 12 August 2016)

\begin{abstract}
Recently, spin excitations in doped cuprates have been measured using resonant inelastic x-ray scattering. The paramagnon dispersions show the large hardening effect in the electron-doped systems and seemingly doping independence in the hole-doped systems, with the energy scales comparable to that of the antiferromagnetic (AFM) magnons. This anomalous hardening effect and the lack of softening were partially explained by using the strong-coupling $t-J$ model but with a three-site term [Nat. Commun. 5, 3314 (2014)], although the hardening effect is already present even without the latter. By considering the $t-t^{\prime}-t^{\prime \prime}-J$ model and using the slave-boson mean-field theory, we obtain, via the spin-spin susceptibility, the spin excitations in qualitative agreement with the experiments. The doping-dependent bandwidth due to the strong correlation physics is the origin of the hardening effect. We also show that dispersions in the AFM regime, different from those in the paramagnetic (PM) regime, hardly vary with dopant density. These excitations are mainly collective in nature instead of particle-hole-like. We further discuss the interplay and different contributions of these two kinds of excitations in the PM phase and show that the dominance of the collective excitation increases with decreasing dopant concentrations.
\end{abstract}

DOI: 10.1103/PhysRevB.94.075127

\section{INTRODUCTION}

It is generally believed that magnetic interaction may be responsible for the superconductivity in cuprates [1]. Recently, the development of resonant inelastic x-ray scattering (RIXS) [2,3] has enabled experimentalists to measure the spin excitations over a more comprehensive region of the Brillouin zone than the conventional inelastic neutron-scattering (INS) experiments [4]. A large family of both electronand hole-doped materials has been investigated and the spin excitations are reported to resemble the dispersion of the antiferromagnetic (AFM) magnon in the paramagnetic (PM) phase, called the paramagnon [4-13]. While many publications demonstrate the magnetic nature of the paramagnon [4-6,10], it is argued that the analogy with spin waves is only partial [11] and the itinerant nature of this magnetic excitation cannot be ignored $[9,12,14-16]$. The strong flavor of the AFM magnon also reinvigorates an old debate that it may be that the AFM fluctuations, seemingly much more robust, are more important for cuprate superconductivity than strong correlation just as for iron-based superconductors [17].

In addition to the magnetic and itinerant nature of this spin excitation, the anomalous doping dependence of the energy dispersions is very intriguing. Contrary to the notion suggested by the INS experiments, the paramagnon dispersions measured by RIXS are of the similar excitation energy scale among different hole dopings [13]. Moreover, the paramagnons show the anomalously large hardening of the energy dispersions in the electron-doped cuprates [8] while holedoped cuprates do not exhibit much softening as hole concentration increases. This is contrary to the expectation

\footnotetext{
*wjli@gate.sinica.edu.tw

†tklee@phys.sinica.edu.tw
}

that paramagnon dispersion will soften when there are more itinerant carriers involved in screening as shown in Ref. [18]. Without including the strong correlation effect on the charges, the spin susceptibility calculated for the $t-J$ model shows significant softening as hole density increases. On the other hand, Jia et al. [19] study an effective single-band Hubbard model using the determinant quantum Monte Carlo and obtain results consistent with experiments. To explain the physics of the hardening effect in electron-doped systems, they introduce a three-site exchange term in a $t-J$-type model. Although their 18-site exact diagonalization calculations including the three-site exchange term do reproduce the correct scale of the hardening effect, their results also show that the hardening effect appears even before their introduction of this extra term. This indicates that the hardening effect is intrinsic in the strong correlation picture of $t-J$ type models without adding any extra interaction terms. Furthermore, they did not consider the different nature of excitations in AFM and PM phases, which can result in different doping dependences of dispersions.

In this work, we would like to point out that these anomalies are signatures of the strong correlation. More precisely, the Mott physics provides a strong bandwidth renormalization as shown by using Gutzwiller approximation (GW) to treat the constraint of no doubly occupied sites in the $t-J$ model [20]. To illustrate this idea in the simplest possible way, we shall use slave-boson $(\mathrm{SB})$ theory $[21,22]$ to include the strong correlation effect. Investigating the model in AFM, PM, and superconducting (SC) phases, we calculate the spin-spin susceptibility and recognize that it is the enhancement of the bandwidth with the dopant density in the PM and SC phases that hardens the energy dispersion, a result of Mott physics accounting for the anomalous experimental observations. The dispersions in AFM phases are only weakly dependent on the dopant density due to their collective nature. Furthermore, 
based on our calculations, we argue that the experimentally observed spin excitations are mixtures of both particle-holelike and paramagnonlike excitations. These mixtures are doping dependent. It is noted that a recent work [16] applied similar methods to the calculations of the Raman spectra of doped cuprates and their results are consistent with some of our conclusions.

\section{THEORETICAL MODEL}

\section{A. AFM and PM phases}

The $t-t^{\prime}-t^{\prime \prime}-J$ model Hamiltonian is written as

$$
\begin{aligned}
H= & -t \sum_{<i j>\sigma}\left(c_{i \sigma}^{\dagger} c_{j \sigma}+\text { H.c. }\right)-t^{\prime} \sum_{<i j>2 \sigma}\left(c_{i \sigma}^{\dagger} c_{j \sigma}+\text { H.c. }\right) \\
& -t^{\prime \prime} \sum_{<i j>3 \sigma}\left(c_{i \sigma}^{\dagger} c_{j \sigma}+\text { H.c. }\right)+J \sum_{<i j>\sigma}\left(\mathbf{S}_{i} \cdot \mathbf{S}_{j}-\frac{1}{4} n_{i} n_{j}\right) \\
& -\mu_{0} \sum_{i \sigma} c_{i \sigma}^{\dagger} c_{i \sigma},
\end{aligned}
$$

where $<>,<>_{2}$, and $<>_{3}$ represent the nearest neighbor, second-nearest neighbor, and third-nearest neighbor, respectively. In the presence of strong Coulomb repulsion, each site is at most singly occupied. As a well-known method to include this constraint, we treat the Hamiltonian by the SB mean-field theory [23,24], i.e., $c_{i \sigma}=b_{i}^{\dagger} f_{i \sigma}$ and $\mathbf{S}_{i}=\frac{1}{2} \sum_{\sigma \sigma^{\prime}} f_{i \sigma}^{\dagger} \boldsymbol{\tau}^{\sigma \sigma^{\prime}} f_{i \sigma^{\prime}}$, with $\boldsymbol{\tau}^{\sigma \sigma^{\prime}}$ the Pauli matrices, $f_{i \sigma}$ the fermionic spinon operator, and $b_{i}$ the bosonic holon operator. The constraint of no double occupancy, $b_{i}^{\dagger} b_{i}+\sum_{\sigma} f_{i \sigma}^{\dagger} f_{i \sigma}=1$, is imposed at each site. Taking the mean-field parameters as $m=(-1)^{i}\left\langle S_{i}^{z}\right\rangle$, the AFM order, and $X=\left\langle f_{i \sigma}^{\dagger} f_{j \sigma}\right\rangle$, the uniform hopping term, the Hamiltonian is written in the momentum space with bosonic operators being replaced by the square root of the average hole density:

$$
\begin{aligned}
H= & \sum_{k, \sigma}{ }^{\prime}\left(\epsilon_{k} f_{k \sigma}^{\dagger} f_{k \sigma}+\epsilon_{k+Q} f_{k+Q \sigma}^{\dagger} f_{k+Q \sigma}\right) \\
& -2 J m \sum_{k, \sigma}{ }^{\prime} \sigma\left(f_{k \sigma}^{\dagger} f_{k+Q \sigma}+\text { H.c. }\right)+2 N J\left(X^{2}+m^{2}\right),
\end{aligned}
$$

where $\sum_{k}{ }^{\prime}$ indicates that the summation is over the magnetic Brillouin zone (MBZ): $-\pi<k_{x} \pm k_{y} \leqslant$ $\pi, \epsilon_{k}=(-2 t \delta-J X)\left(\cos k_{x}+\cos k_{y}\right)-4 t^{\prime} \delta \cos k_{x} \cos k_{y}-$ $2 t^{\prime \prime} \delta\left(\cos 2 k_{x}+\cos 2 k_{y}\right)-\mu, Q=(\pi, \pi)$, and $N$ is the total number of lattice sites. Due to the strong correlation between electrons, the hopping terms of the energy bands are modulated by the dopant density, which is similar to the Gutzwiller approximation to replace the constraint of forbidding double occupancy with a renormalization factor, $g_{t}=2 \delta /(1+\delta)$ [20]. Here our factor is about two times smaller, which will be discussed later.

By taking the unitary transformations $f_{k \sigma}=\cos \theta_{k} \alpha_{k \sigma}+$ $\sigma \sin \theta_{k} \beta_{k \sigma}$ and $f_{k+Q \sigma}=-\sigma \sin \theta_{k} \alpha_{k \sigma}+\cos \theta_{k} \beta_{k \sigma} \quad$ with $\cos 2 \theta_{k}=\left(\epsilon_{k+Q}-\epsilon_{k}\right) / \gamma_{k}, \quad \sin 2 \theta_{k}=-4 J \mathrm{~J} / \gamma_{k}$, and $\gamma_{k}=$
$\sqrt{\left(\epsilon_{k+Q}-\epsilon_{k}\right)^{2}+(4 J m)^{2}}$, we obtain

$$
H=\sum_{k, \sigma}{ }^{\prime}\left(\xi_{k \alpha} \alpha_{k \sigma}^{\dagger} \alpha_{k \sigma}+\xi_{k \beta} \beta_{k \sigma}^{\dagger} \beta_{k \sigma}\right)+2 N J\left(X^{2}+m^{2}\right),
$$

with the energy bands $\xi_{k \alpha, \beta}=\left(\epsilon_{k}+\epsilon_{k+Q} \mp \gamma_{k}\right) / 2$. The free energy is given by $F=-2 T \sum_{\eta=\alpha, \beta} \sum_{k}^{\prime} \ln \left(1+e^{-\xi_{k \eta} / T}\right)+$ $2 N J\left(X^{2}+m^{2}\right)$. The mean-field parameters $m$ and $X$, as well as the chemical potential $\mu$, are determined self-consistently by the conditions $\partial F / \partial m=0, \partial F / \partial X=0$, and $-(\partial F / \partial \mu)=$ $N(1-\delta)$ at zero temperature. Based on these mean-fielddetermined parameters, we calculate the spin susceptibility. The model parameters taken throughout the work are $t=1.0$, $t^{\prime}=-0.3, t^{\prime \prime}=0.2$, and $J=0.3$ for the hole-doped case, while $t^{\prime}=0.3$ and $t^{\prime \prime}=-0.2$ for the electron-doped case [25].

The transverse spin susceptibility is defined as

$$
\chi_{(0)}^{ \pm}\left(q, q^{\prime}, \tau\right)=\frac{1}{N}\left\langle T_{\tau} S_{q}^{+}(\tau) S_{-q^{\prime}}^{-}(0)\right\rangle_{(0)},
$$

where $\langle\cdots\rangle_{0}$ means the thermal average on the eigenstates of the mean-field Hamiltonian, $S_{q}^{+}=\sum_{i} S_{i}^{+} e^{i q \cdot R_{i}}$, and $S_{q}^{-}=$ $\left(S_{-q}^{+}\right)^{\dagger}$. The residual fluctuation of the spin-spin interaction is taken into account by the random phase approximation (RPA) [24].

Because of the nonvanishing off-diagonal correlation function as a result of antiferromagnetism, the spin susceptibility is written as a matrix:

$$
\hat{\chi}^{ \pm}=\left(\begin{array}{cc}
\chi^{ \pm}\left(q, i \omega_{n}\right) & \chi^{ \pm}\left(q, q+Q, i \omega_{n}\right) \\
\chi^{ \pm}\left(q+Q, q, i \omega_{n}\right) & \chi^{ \pm}\left(q+Q, i \omega_{n}\right)
\end{array}\right) .
$$

The diagonal term is given as

$$
\begin{aligned}
\chi_{0}^{ \pm}\left(q, i \omega_{n}\right)= & -\frac{1}{N} \sum_{k}^{\prime}\left[\cos ^{2}\left(\theta_{k}+\theta_{k+q}\right)\left(F_{\alpha \alpha}+F_{\beta \beta}\right)\right. \\
& \left.+\sin ^{2}\left(\theta_{k}+\theta_{k+q}\right)\left(F_{\alpha \beta}+F_{\beta \alpha}\right)\right],
\end{aligned}
$$

and the off-diagonal term is given as

$$
\begin{aligned}
\chi_{0}^{ \pm}\left(q, q+Q, i \omega_{n}\right) \\
=\frac{1}{2 N} \sum_{k}^{\prime}\left[\left(\sin 2 \theta_{k+q}-\sin 2 \theta_{k}\right)\left(F_{\alpha \alpha}-F_{\beta \beta}\right)\right. \\
\left.\quad+\left(\sin 2 \theta_{k+q}+\sin 2 \theta_{k}\right)\left(F_{\alpha \beta}-F_{\beta \alpha}\right)\right],
\end{aligned}
$$

with the abbreviations

$$
F_{\eta \eta^{\prime}}=\frac{n\left(\xi_{k+q, \eta}\right)-n\left(\xi_{k, \eta^{\prime}}\right)}{i \omega_{n}+\xi_{k+q, \eta}-\xi_{k, \eta^{\prime}}}\left(\eta, \eta^{\prime}=\alpha, \beta\right) .
$$

$n(z)=1 /\left(1+e^{(z / T)}\right)$ is the Fermi function, and $i \omega_{n}$ are the Matsubara frequencies. The RPA result is given as

$$
\hat{\chi}_{\mathrm{RPA}}^{ \pm}=\hat{\chi}_{0}^{ \pm}\left[I+\hat{\chi}_{0}^{ \pm} \hat{J}\right]^{-1},
$$

where $I$ is the identity matrix and

$$
\hat{J}=\left(\begin{array}{cc}
J(q) & 0 \\
0 & J(q+Q)
\end{array}\right),
$$

with $J(q)=J\left(\cos q_{x}+\cos q_{y}\right)$. We can see from the equations above that there are two parts contributing to the spin-spin excitations. One is the particle-hole excitation constituted of the interband ( $\alpha$ to $\beta$ or $\beta$ to $\alpha$ ) and the intraband ( $\alpha$ to $\alpha$ or $\beta$ to 
$\beta$ ) excitations, which are described by $\chi_{0}^{ \pm}$, or more specifically the term $F_{\eta \eta^{\prime}}$. The other is the collective spin-wave excitation mode, which is a result of the additional poles generated by the RPA calculation from $\operatorname{det}\left[I+\hat{\chi}_{0}^{ \pm} \hat{J}\right]=0$.

For the paramagnetic case, the off-diagonal term of the spin susceptibility vanishes and the result is simply given as

$$
\chi_{0}^{ \pm}\left(q, i \omega_{n}\right)=-\frac{1}{N} \sum_{k} \frac{n\left(\epsilon_{k+q}\right)-n\left(\epsilon_{k}\right)}{i \omega_{n}+\epsilon_{k+q}-\epsilon_{k}},
$$

and

$$
\chi_{\mathrm{RPA}}^{ \pm}\left(q, i \omega_{n}\right)=\frac{\chi_{0}^{ \pm}\left(q, i \omega_{n}\right)}{1+\chi_{0}^{ \pm}\left(q, i \omega_{n}\right) J(q)} .
$$

In this case, usually the denominator does not have a sharp pole. Therefore the numerator with particle-hole excitation becomes dominant at some $q$, where the weights of $\chi_{0}^{ \pm}$are only slightly modified by the denominator in the RPA calculation.

\section{B. Superconducting phase}

In the SC phase [26-32], the mean-field Hamiltonian is obtained by decoupling the spin-spin interaction term $\mathbf{S}_{i} \cdot \mathbf{S}_{j}$ into pairing and direct hopping terms [30], and choosing the mean-field parameters $\Delta_{i j}=\left\langle f_{i \uparrow} f_{j \downarrow}-f_{i \downarrow} f_{j \uparrow}\right\rangle= \pm \Delta_{0}$, $X_{0}=\sum_{\sigma}\left\langle f_{i \sigma}^{\dagger} f_{j \sigma}\right\rangle$, and $\left\langle b_{i}\right\rangle=\sqrt{\delta}$ :

$$
\begin{aligned}
H= & \sum_{k, \sigma} \xi_{k} f_{k \sigma}^{\dagger} f_{k \sigma}-\sum_{k} \Delta_{k}\left(f_{k \uparrow}^{\dagger} f_{-k \downarrow}^{\dagger}+\text { H.c. }\right) \\
& +2 N J\left(X_{0}^{2}+\Delta_{0}^{2}\right),
\end{aligned}
$$

where $\quad \xi_{k}=\left(-2 \delta t-2 J X_{0}\right)\left(\cos k_{x}+\cos k_{y}\right)-4 \delta t^{\prime} \cos k_{x}$ $\cos k_{y}-2 \delta t^{\prime \prime}\left(\cos 2 k_{x}+\cos 2 k_{y}\right)-\mu \quad$ and $\Delta_{k}=2 J^{\prime} \Delta_{0}$ $\left(\cos k_{x}-\cos k_{y}\right)$, with $J^{\prime}=3 J / 8$. Here $X_{0}$ includes hoppings of both spins.

The spin-spin susceptibility applying RPA is given by Eq. (11), with the numerator

$$
\chi_{0}^{ \pm}\left(q, i \omega_{n}\right)=-\frac{1}{N} \sum_{k ; \eta, \eta^{\prime}= \pm} C_{\eta \eta^{\prime}} \frac{n\left(\eta E_{k+q}\right)-n\left(\eta^{\prime} E_{k}\right)}{i \omega_{n}+\eta E_{k+q}-\eta^{\prime} E_{k}}
$$

where $E_{k}=\sqrt{\xi_{k}^{2}+\Delta_{k}^{2}}$ is the quasiparticle excitation energy in the SC state. The coefficients $C_{\eta \eta^{\prime}}$ are given as follows: $C_{++}=v_{k+q}^{2} v_{k}^{2}+u_{k+q} v_{k+q} u_{k} v_{k}, C_{--}=u_{k+q}^{2} u_{k}^{2}+$ $u_{k+q} v_{k+q} u_{k} v_{k}, C_{+-}=v_{k+q}^{2} u_{k}^{2}-u_{k+q} v_{k+q} u_{k} v_{k}$, and $C_{-+}=$ $u_{k+q}^{2} v_{k}^{2}-u_{k+q} v_{k+q} u_{k} v_{k}$, with the coherence factors $v_{k}^{2}=$ $\frac{1}{2}\left(1-\frac{\xi_{k}}{E_{k}}\right), u_{k}^{2}=\frac{1}{2}\left(1+\frac{\xi_{k}}{E_{k}}\right)$, and $u_{k} v_{k}=\frac{\Delta_{k}}{2 E_{k}}$.

\section{RESULTS AND COMPARISON WITH EXPERIMENTS}

The imaginary part of the spin susceptibility reflects the possible excitations, identified by the peaks, and their weights. In Fig. 1, as an example, we show the imaginary part of $\chi_{\mathrm{RPA}}^{ \pm}$ in AFM cases [40], along different paths in the momentum space with analytical continuation $i \omega_{n} \rightarrow \omega+i \Gamma$ performed. We take the damping parameter $\Gamma=0.01|t|$ and $500 \times 500$ $k$ points in the first Brillouin zone (or half of the $500 \times 500$ $k$ points in the MBZ) in all of our calculations. The energy spread of the excitation is related to the bandwidth, which is roughly proportional to the dopant density. Accordingly, the

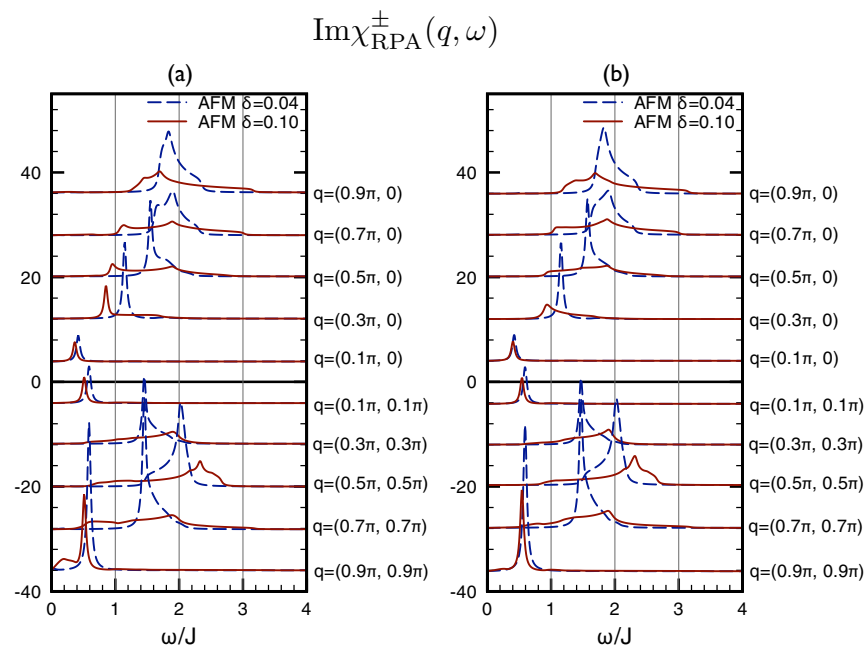

FIG. 1. The imaginary part of the spin-spin susceptibility in the AFM phase along different momentum paths of (a) hole-doped and (b) electron-doped cases. The dashed lines and the solid lines represent $\delta=0.04$ and 0.10 , respectively. The values in both cases of $q=(0.9,0.9) \pi$ are reduced by 10 ; while $q=(0.7,0.7) \pi$ and $(0.5,0.5) \pi$ are reduced by 2 , in order to fit into the figure.

excitation spectrum broadens as the dopant density increases. Also, as the momentum $q$ gets larger along the $(\pi, 0)$ direction (the upper panels in Fig. 1) or gets closer to $\pi / 2$ along the $(\pi, \pi)$ direction (the lower panels in Fig. 1), the spectrum broaden as a result of the broader range of the accessible particle-hole excitation energies. This is an example showing that, in addition to the spin-wave excitations (the local picture), particle-hole excitations (the itinerant picture) also contribute to the spin susceptibility away from the resonant $k$ points. The detailed situation depends on the dopant density but this general feature of the mixing of two types of excitations is prevailing throughout this work. We will discuss this below.

From Eqs. (8) and (11), the particle-hole-like excitations appear when the numerators dominate while the paramagnonlike excitations show up with larger spectral weight due to the vanishing of the denominator. In the low-doping (AFM) regime, the resonance from the denominator is sharp and hardly doping dependent while in the PM phase it becomes smooth and the contribution from the numerator becomes significant. In order to demonstrate it more clearly, we separate the total spin susceptibility into particle-holelike and paramagnonlike contributions (see Appendix A for details). In Fig. 2, the spin susceptibility of a PM $e$-doped system with $\delta=0.15$ is calculated along both $(\pi, 0)$ and $(\pi, \pi)$ directions. The total susceptibility (green lines) can be decomposed into contributions from the inverse of the denominator or paramagnon (red lines) and the numerator or particle-hole excitations (black lines). Along both directions, the contribution from the denominator dominates at low $q$ and at around the AFM point $(\pi, \pi)$ while the numerator has larger contributions at other momentum transfer. Larger dopant density corresponds to smaller $q$ range where paramagnon excitations dominate (see Appendix A). However, the general patterns of two types of excitations are similar for all dopings. 


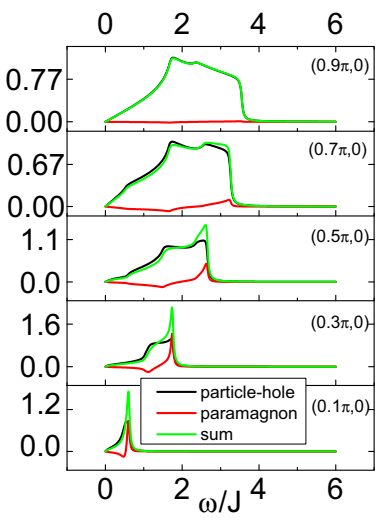

(a)

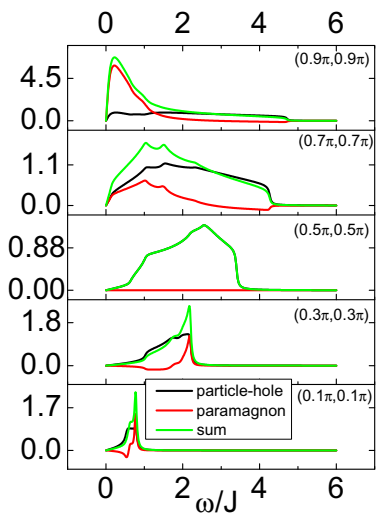

(b)
FIG. 2. The spin susceptibility for the $e$-doped system with $\delta=$ 0.15 . (a) Along the $(\pi, 0)$ direction. (b) Along the $(\pi, \pi)$ direction. At low $q$ and at around the AFM point $(\pi, \pi)$, the peak of the total susceptibility (green) is mostly contributed by the spin-wave-like excitations (red). For other momentum transfer $q$, the feature of the total susceptibility is dominated by the particle-hole-like excitations (black).

Recent RIXS experiments seem to conclude that there are at least two distinct elementary excitations existing in the cuprate superconductors and their appearance depends both on the polarization of the incident photons and on the scattering geometry $[10,15]$. One is the particle-hole like excitation the resonance peaks of which change positions with different incident photon energies. The other one is the paramagnonlike excitation the resonance peaks of which are located independently of the incident photon energy. However, from our calculations we find these two excitations all contribute to the spin susceptibility, which is consistent with the recent experiment [33]. They may have a dispersion similar to AFM spin waves at small momenta $q$, but they are mixed together. This is consistent with recent experiments [9,15] reporting similar excitation energy scales measured using different photon polarizations and scattering geometries.

For every momentum $q$ in the spin susceptibility, we identify the maximum of $\operatorname{Im} \chi_{\mathrm{RPA}}^{ \pm}$as the excitation energy. We plot the excitation energies with different momenta, thus the dispersion relation of the spin excitation, in Fig. 3 for different hole concentrations in (a) and electrons in (b). The spin susceptibility is not symmetric about the peak so the half width at half maximum (HWHM) is not well-defined here. Therefore, we indicate the distance from the peak to the half maximum of the susceptibility as the error bars and the error bars on the high-energy (right) side of the peak can be different from those on the low-energy (left) side. In the high momentum region near $q=(\pi, 0)$ and $(\pi / 2, \pi / 2)$, the broadness of the spectrum makes the identification of the excitation energy difficult and causes large fluctuations as well as the feature of particle-hole excitations mentioned above.

In AFM cases, the dispersions are the collective spin-wave mode excitations, which agrees with experiments. [4-6] The dispersion at small $q$ does not change significantly with the dopant density in the AFM phase, which points out the collective nature of the AFM excitations determined mainly by

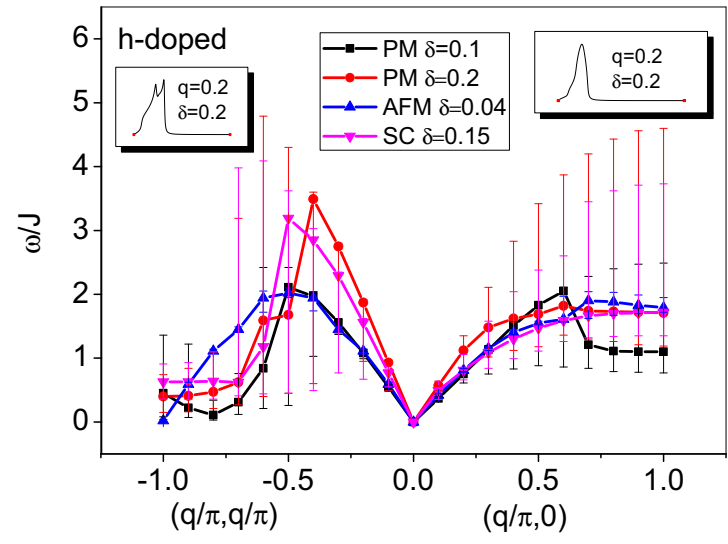

(a)

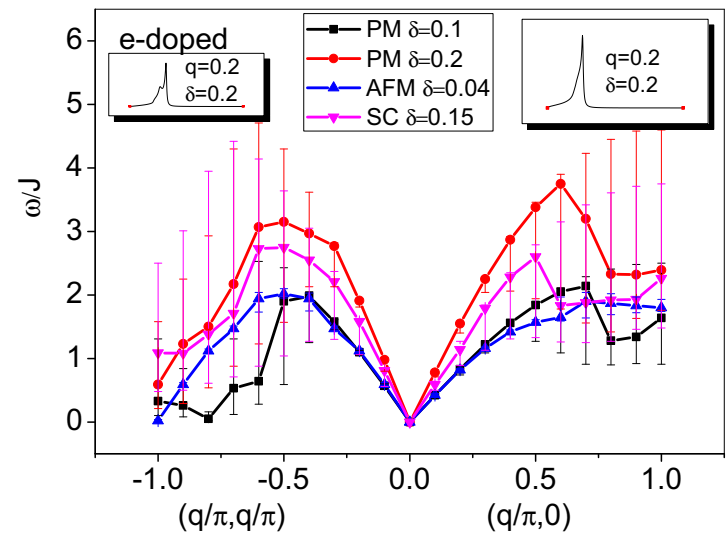

(b)

FIG. 3. The dispersion relation of the spin excitations in AFM and PM (a) of the hole-doped case and (b) of the electron-doped case. The ends of the error bars indicate the half-maximum points. The points at the zero momentum are artificial in order to visualize the linearity. The electron-doped systems show the hardening effects along both directions while the hardening effect in the hole-doped systems along the $(\pi, 0$,$) direction is reduced. Insets are the spin susceptibilities with$ (electron or hole) doping equal to 0.2 and momentum transfer $q=0.2$ in both directions in $k$ space.

the minima of the denominator. The AFM result for $\delta=0.1$ is quite similar to the PM dispersion shown in Fig. 3(b), hence it is not shown. The difference between the AFM results of $\delta=0.1$ and 0.04 is only noticeable at large $q$, confirming the weak dopant-density dependence in the AFM phase. The dispersions for each doping are similar for the PM and SC cases. For clarity, we only show $\delta=0.1$ and 0.2 for the PM phase and $\delta=0.15$ for the SC phase. Note that in Fig. 3(a) a much narrowed dispersion around $(\pi, \pi)$ for the SC phase at $\delta=0.15$ is shown. This is consistent with the neutron-scattering result and our previous calculations [26].

In both electron-doped and hole-doped cases, the dispersion relation is linear for small $q$. The slope of excitations at small $q$ increases with doping significantly for electron doped cases as shown in Fig. 3(b). This hardening effect was observed in recent experiments [8]. Along the $q_{x}$ direction for the hole-doped cases, the slope is only slightly dependent on the dopant density [see the right panel of Fig. 3(a)], which is also qualitatively consistent with experiments [4-6,8,11-13,34]. 


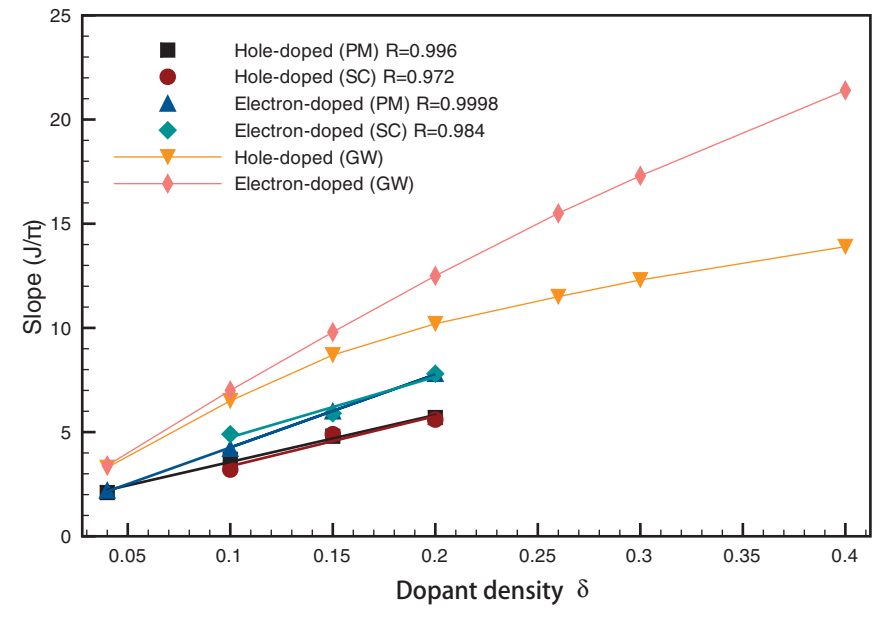

FIG. 4. The slope near zero momentum of the dispersions as a functions of dopant density. The relations are almost linear, with the electron-doped cases ascending faster than the hole-doped cases as the doping increases. The case of Gutzwiller approximation (GW) is also plotted. AFM channels are turned off at all dopant densities to demonstrate the bandwidth effect only.

These doping dependences are due to the doping-dependent bandwidth originated from the strong electron-electron correlations. This will be discussed in the next paragraph. Insets in Fig. 3 are the spin susceptibilities with (electron- or hole-) dopant density $\delta=0.2$ and momentum transfer $q=0.2$ in both directions in $k$ space. Only the hole-doped case along the $(\pi, \pi)$ direction shows a two-peak feature, the possible consequences of which will be discussed in Sec. IV.

Consider $\epsilon_{k}$ in Eq. (2) along the $q_{x}$ direction. It is easy to see that the slope $s$ of the energy dispersion in the long-wavelength region is proportional to the bandwidth. The bandwidth $W_{\pi 0} \sim \epsilon_{(\pi / 2,0)}-\epsilon_{(0,0)}=2 \delta\left(t+2 t^{\prime}+2 t^{\prime \prime}\right)+$ $J \chi$ and $\frac{\Delta s}{\Delta \delta} \sim \frac{d W_{\pi 0}}{d \delta}=2 t+4\left(t^{\prime}+t^{\prime \prime}\right)$. For the electron-doped cases, $t^{\prime}+t^{\prime \prime}$ is positive while $t^{\prime}+t^{\prime \prime}$ is negative for the hole-doped cases. Thus the bandwidth has a larger dependence on dopant density for the electron-doped cases than in the hole-doped cases. Similar analysis can be performed along the $(\pi, \pi)$ direction (see Appendix B). When the superconductivity exists, in addition to the particle-hole excitations, we also need to consider the particle-particle excitations. Estimating $\Delta_{0}$ as 0.2 , the superconducting gap $\Delta_{k} \approx \frac{3 J}{2} \Delta_{0} \approx 0.3 J$, which is small compared to the original band. Therefore, the excitation spectra are not much altered in the presence of the superconducting gap (see Fig. 3), which is consistent with recent experimental observation showing similar excitation dispersions above and below $T_{c}$ [35].

In order to illustrate the effect of the renormalized bandwidth explicitly, we plot the dopant-density-dependent slope of energy dispersion in the small momentum regime along the $(\pi, 0)$ direction in Fig. 4. Note that AFM channels are turned off at all dopant densities in this figure to demonstrate the bandwidth effect only. We also include the GW by multiplying the hopping integrals by $g_{t}=2 \delta /(1+\delta)$. Since the $\mathrm{GW}$ factor is proportional to $2 \delta$ instead of just $\delta$ as in the SB result, the slopes shown in Fig. 4 are about twice larger than that of SB for both hole- and electron-doped cases. This confirms that

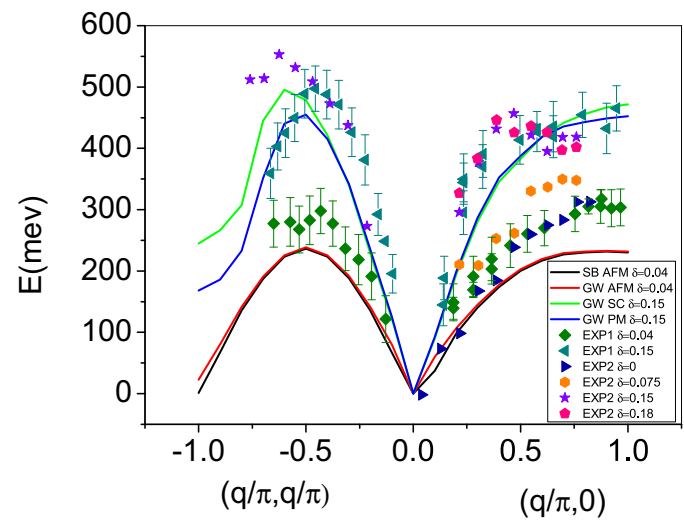

FIG. 5. Comparison of energies of the energy excitations of our calculations with the experiments (EXP1 [8] and EXP2 [34]) of electron-doped NCCO with $J=120 \mathrm{meV}$ and HMHW $=50 \mathrm{meV}$. SB stands for slave-boson method and GW stands for Gutzwiller.

the hardening is due to the band renormalization by the strong correlation. Since experiments for hole-doped systems have found similar dispersions [4-6,11-13] for doping between 10 and $40 \%$ (see the right panel of Fig. 6), the reduced hardening in our hole-doped calculations indicates the strong correlation is still present for doping as large as $40 \%$.

Our results are compared with experiments on the electrondoped cuprates $[8,34]$ in Fig. 5. In addition to the SB, calculations with $\delta$ replaced by the GW factor $2 \delta /(1+\delta)$ are also carried out. The enhanced hardening effect in the experimental data is well reproduced by both the SB method (in AFM cases) and GW approximations (all dopings). This consistency with experimental observations is quite surprising as we have not included the core hole effect [2]. Also, this provides a physical insight on the hardening effect before including the three-site term in the $t-J$ model [19]. Note that due to the collective nature of the AFM excitations both methods give similar results even though the bandwidth in the GW method is nearly twice that of the SB method.

In Fig. 6, we compare our results with hole-doped experiments along the $(\pi, 0)$ direction $[4-6,9,11-13,15]$ and along

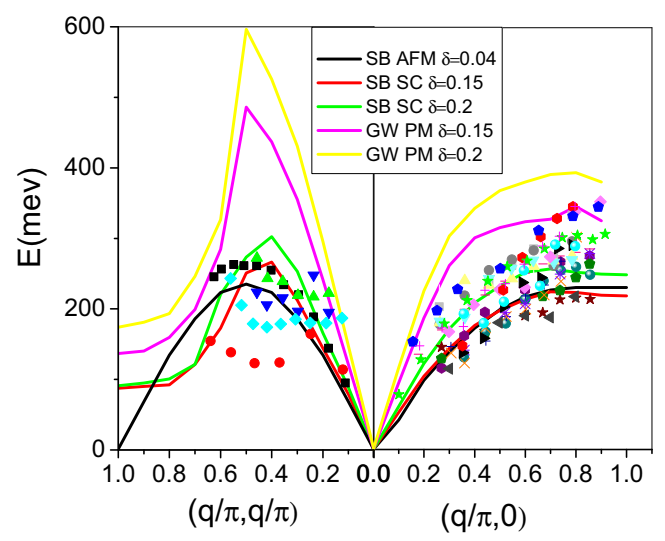

FIG. 6. Comparison of excitations of our calculations using $J=$ $120 \mathrm{meV}$ with experiments of hole-doped systems [4-6,9,11-13,15] and $\mathrm{HMHW}=50 \mathrm{meV}$. SB stands for slave-boson method and GW stands for Gutzwiller. 
the $(\pi, \pi)$ direction $[9,11,15]$. For the $(\pi, 0)$ direction, our results are consistent with peaks and line shapes reported by experiments and also having a similar energy spread. Along the $(\pi, \pi)$ direction, our dispersion using the GW factors is less consistent with experiments. One possible reason is that it is difficult to determine the peak position as there are two peaks as shown in the left inset in Fig. 3. This issue will be discussed more in the next section. All of our results are shown after applying the Gaussian convolution (see Appendix C); the half width at half maximum of the distribution is $50 \mathrm{meV}$ for Figs. 5 and 6.

\section{DISCUSSIONS}

Jia et al. [19] pointed out that while the hardening of the $e$ doped systems can be well explained by $t-J$ like models the hole-doped cases are not well fitted and need the considerations of the full Hubbard model. The situation is similar with our calculation. While the reduced hardening effect along the $(\pi, 0)$ direction in the hole-doped calculations is qualitatively consistent with the excitation spectrum in the experiments, the remarkable hardening effect along the $(\pi, \pi)$ direction is different from the experimental observations $[9,11,15]$, indicating some key ingredients missed for this case in the simple SB+RPA calculations.

In order to get more insights on this issue, we study the line shape of the spin susceptibility in detail. We find that most line shapes are of a well-defined one-peak structure while the two-peak structure appears in hole-doped systems along $(\pi, \pi)$ direction, as shown in insets in Fig. 3. Because these two peak values are close, upon introducing extra interactions or considering other possible effects, the larger peak of the two, defined as the excitation peak, may switch while those susceptibilities with one-peak structure are relatively robust. This may change our calculated excitation dispersions.

As an example, we consider a frequency-dependent lifetime $\tau(\omega)$ of quasiparticles $(1 / \tau \sim a+b \omega$ in the marginal-Fermiliquid theory [36] and $1 / \tau \sim c+d \omega^{2}$ in the normal-Fermiliquid theory) in the mean-field SB stage. The inclusion of this variable lifetime switches some of the maxima of those spin susceptibilities with two-peak structures and leads to the nearly doping-independent excitation spectrum in the hole-doped cases along the $(\pi, \pi)$ direction (doping $=0.15-0.2$ ) while other cases [electron-doped systems and hole-doped systems along the $(\pi, 0)$ direction] are almost unchanged by this inclusion.

Although only partially consistent with experiments, our theory does show the uniqueness of the hole-doped systems along the $(\pi, \pi)$ direction. We argue that by including some minor interactions or effects, which is out of the scope of our simple SB+RPA scheme here, the excitation dispersion for hole-doped systems along the $(\pi, \pi)$ direction can be modified and reach better consistency with experimental observations.

In general, the SB theory is the simplest approach to incorporate the Mott physics about charge degree of freedom. It strongly renormalizes quasiparticle spectral weight by the doping density $\delta$. It ensures an insulator without doping. Combined with RPA, this approach captures the corresponding spin dynamics. Although we expect SB+RPA to be only qualitatively accurate, surprisingly the results are semiquantitatively consistent with experimental observations. Several factors may cause the quantitative discrepancies in our SB+RPA calculations. As discussed in Ref. [37], a full SB theory contains four Boson fields instead of only one in our case. Also, it is possible that the RIXS measurements only qualitatively agree with the spin dynamic structure factor, as mentioned by a very recent theoretical work [38]. Furthermore, the core effect and detailed spin-flipping processes have to be included in the full treatment of the Kramers-Heisenberg theory (as was done in Ref. [19]), whose quantitative agreements with experiments cannot be achieved by a simple SB+RPA theory. Therefore, we need sophisticated theories for fully quantitative predictions but our work is good for understanding the hardening physics of RIXS in cuprates.

\section{CONCLUSIONS}

In summary, we investigate the spin-spin susceptibility in the $t-t^{\prime}-t^{\prime \prime}-J$ model, via SB mean-field theory. The excitation spectra are determined through the peaks of the imaginary part of the susceptibility. The paramagnon hardening effect, consistent with experimental observations in electron-doped cuprates, comes from the doping dependent bandwidth, revealing the strong correlation. The dispersions in AFM phases are only weakly dopant dependent due to their collective nature. Nevertheless, the hardening effect is lessened in the hole-doped materials, partly reflecting the nearly doping-independent energy dispersion in hole-doped experiments. We argue that discrepancies in the hole-doped systems along the $(\pi, \pi)$ direction may be reduced by including some minor interactions or effects. We also show that both particle-hole-like and collective paramagnonlike excitations are usually coupled together and not easily separated. The range where the collective excitations dominate increases with decreasing dopant concentrations in the PM phase. The increase of bandwidth with dopant density due to the strong correlation is still present over a wide doping range in cuprates.

\section{ACKNOWLEDGMENTS}

The authors acknowledge useful discussions with Dr. SungPo Chao (Academia Sinica, Taiwan). In particular, we would like to thank Dr. Wei-Sheng Lee (Stanford Linear Accelerator Center, a National Accelerator Laboratory) for sharing with us some of the experimental data before it was published and also for many stimulating discussions. W.-J.L., C.-J.L., and T.-K.L. acknowledge support by Ministry of Science and Technology (MOST) in Taiwan (Grant No. 104-2112-M-001-005).

\section{APPENDIX A: THE PARTICLE-HOLE AND COLLECTIVE EXCITATIONS IN AN ELECTRON-DOPED SYSTEM WITH A LOW DOPING $(\delta=0.04)$}

The total susceptibility is separated into particle-hole-like and paramagnonlike contributions, denoted as $\chi_{\mathrm{ph}}^{ \pm}$and $\chi_{\text {para }}^{ \pm}$, respectively. These two contributions can be defined based on Eq. (11) as follows:

$$
\chi_{\text {para }}^{ \pm}=\operatorname{Re}\left[\chi_{0}^{ \pm}\left(q, i \omega_{n}\right)\right] \times \operatorname{Im}\left(\frac{1}{1+\chi_{0}^{ \pm}\left(q, i \omega_{n}\right) J(q)}\right),
$$




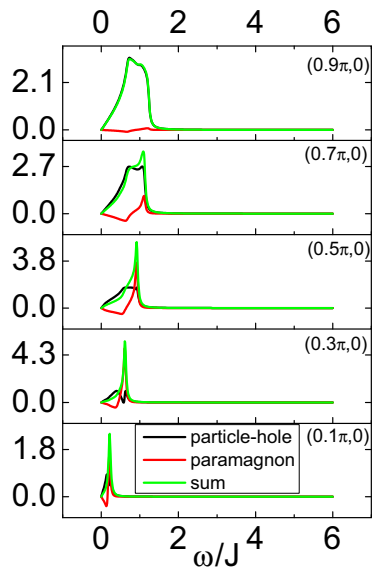

(a)

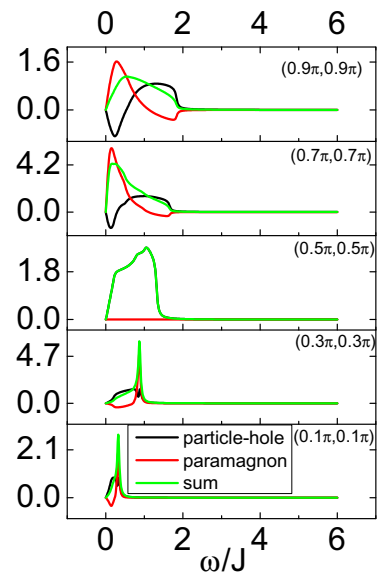

(b)
FIG. 7. The spin susceptibility for the $e$-doped system with $\delta=0.04$. (a) Along the $(\pi, 0)$ direction. (b) Along the $(\pi, \pi)$ direction. Compared to the case with $\delta=0.15$ discussed in the main text, the total susceptibility (green) is more dominant by the spin-wave-like excitations (red) than the particle-hole like excitations (black). For $q=(0.9 \pi, 0.9 \pi)$, the particle-hole contribution is not small, which might be due to the unrealistic PM phase in this low-doping case.

$$
\chi_{\mathrm{ph}}^{ \pm}=\operatorname{Im}\left[\chi_{0}^{ \pm}\left(q, i \omega_{n}\right)\right] \times \operatorname{Re}\left(\frac{1}{1+\chi_{0}^{ \pm}\left(q, i \omega_{n}\right) J(q)}\right),
$$

where $\operatorname{Re}(a)$ and $\operatorname{Im}(a)$ denote real and imaginary parts of $a$, respectively. In the main text we examine the PM system with $\delta=0.15$. In order to further examine the doping dependence of $\chi_{\mathrm{ph}}^{ \pm}$and $\chi_{\mathrm{para}}^{ \pm}$, the AFM channel is turned off for obtaining the PM cases with a low doping $\delta=0.04$, as shown in Fig. 7. The collective excitations are more dominant, compared to the high doping ( $\delta=0.15$ ) cases in the main text.

\section{APPENDIX B: EFFECTS OF $t^{\prime}$ AND $t^{\prime \prime}$}

The combination of $t^{\prime}$ and $t^{\prime \prime}$ causes the asymmetry between electron-doped and hole-doped systems. In Fig. 8, the doping dependence of the dispersion slope is calculated by linearly fitting the low- $q$ regime of the excitation spectrum with different sets of $\left\{t, t^{\prime}, t^{\prime \prime}\right\}$. The hopping constants are the same as those taken in the main text when they are chosen to be turned on. The general feature is that they all have the hardening effect. The asymmetry between electron-doped and hole-doped systems is present upon turning on $t^{\prime}$ and/or $t^{\prime \prime}$. For hole-doped cases, $t^{\prime}$ and $t^{\prime \prime}$ do not provide much qualitative

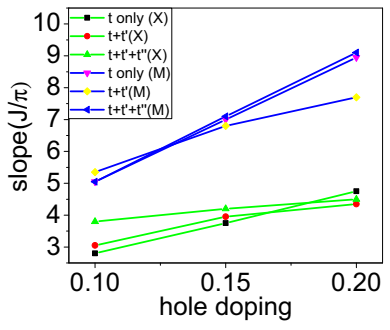

(a)

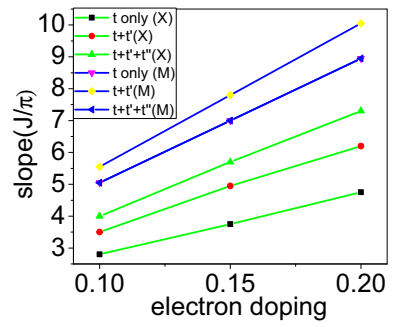

(b)
FIG. 8. The effects of $t^{\prime}$ and $t^{\prime \prime}$ on the low- $q$ slope of the spin excitation spectrum (a) of the hole-doped case and (b) of the electrondoped case. X (green lines) denotes the $(\pi, 0)$ direction while M (blue lines) denotes the $(\pi, \pi)$ direction. They all show the hardening effect. The asymmetry between the electron-doped and hole-doped systems occurs with the inclusion of $t^{\prime}$ and/or $t^{\prime \prime}$.

or quantitative difference. For $e$-doped cases, hardening is always observed, but $t^{\prime}$ and $t^{\prime \prime}$ seem to enhance it a little bit. By performing similar calculations to those in the main text, the bandwidth along the $(\pi, \pi)$ direction $W_{\pi \pi} \sim \epsilon_{(\pi / 2, \pi / 2)}-\epsilon_{(0,0)}$ and $\frac{d W_{\pi \pi}}{d \delta}=4 t+4\left(t^{\prime}+2 t^{\prime \prime}\right)$. Combined with the formula of $\frac{d W_{\pi 0}}{d \delta}$ in the main text, the effect of $t^{\prime}$ and $t^{\prime \prime}$ can be qualitatively explained. Although these results are obtained using RPA after the mean-field calculations, an analysis in the mean-field level (band renormalization by the Mott physics) can catch the main features of these results.

\section{APPENDIX C: GAUSSIAN CONVOLUTION}

Due to the limited energy resolution in some of the RIXS experiments [3], we have to apply the Gaussian convolution to our calculation results before comparing them to the experimental observations. Gaussian function is defined as

$$
G(\omega)=\exp \left(-\frac{\omega^{2}}{2 \sigma}\right)
$$

For every frequency $\omega$, the newly convoluted data are calculated by

$$
\text { new data }(\omega)=A \frac{\sum_{n} G\left(\omega-\omega_{n}\right) \times \text { original data }\left(\omega_{n}\right)}{\sum_{n} G\left(\omega-\omega_{n}\right)},
$$

where $n$ stands for summing all the frequency points, and $A$ is a normalization factor to keep the total weight conserved. As an example, suppose HWHM in the RIXS experiments is around $50 \mathrm{meV}$. The HWHM is given by $\sqrt{2 \ln 2 \sigma}$ so that we set $\sigma=0.354 J$ with $J=120 \mathrm{meV}$ in the Gaussian convolutions.
[1] P. A. Lee, N. Nagaosa, and X.-G. Wen, Rev. Mod. Phys. 78, 17 (2006).

[2] L. J. P. Ament, M. van Veenendaal, T. P. Devereaux, J. P. Hill, and J. van den Brink, Rev. Mod. Phys. 83, 705 (2011).

[3] M. Dean, J. Magn. Magn. Mater. 376, 3 (2015).

[4] M. Le Tacon, G. Ghiringhelli, J. Chaloupka, M. M. Sala, V. Hinkov, M. W. Haverkort, M. Minola, M. Bakr, K. J. Zhou,
S. Blanco-Canosa, C. Monney, Y. T. Song, G. L. Sun, C. T. Lin, G. M. De Luca, M. Salluzzo, G. Khaliullin, T. Schmitt, L. Braicovich, and B. Keimer, Nat. Phys. 7, 725 (2011).

[5] M. Le Tacon, M. Minola, D. C. Peets, M. Moretti Sala, S. BlancoCanosa, V. Hinkov, R. Liang, D. A. Bonn, W. N. Hardy, C. T. Lin, T. Schmitt, L. Braicovich, G. Ghiringhelli, and B. Keimer, Phys. Rev. B 88, 020501 (2013). 
[6] M. P. M. Dean, G. Dellea, R. S. Springell, F. Yakhou-Harris, K. Kummer, N. B. Brookes, X. Liu, Y.-J. Sun, J. Strle, T. Schmitt, L. Braicovich, G. Ghiringhelli, I. Božović, and J. P. Hill, Nat. Mater. 12, 1019 (2013).

[7] L. Braicovich, J. van den Brink, V. Bisogni, M. M. Sala, L. J. P. Ament, N. B. Brookes, G. M. De Luca, M. Salluzzo, T. Schmitt, V. N. Strocov, and G. Ghiringhelli, Phys. Rev. Lett. 104, 077002 (2010).

[8] W. S. Lee, J. Lee, E. Nowadnick, S. Gerber, W. Tabis, S. Huang, V. N. Strocov, E. M. Motoyama, G. Yu, B. Moritz, H. Huang, R. Wang, Y. Huang, W. Wu, C. Chen, D. Huang, M. Greven, T. Schmitt, Z. X. Shen, and T. P. Devereaux, Nat. Phys. 10, 883 (2014).

[9] S. Wakimoto, K. Ishii, H. Kimura, M. Fujita, G. Dellea, K. Kummer, L. Braicovich, G. Ghiringhelli, L. M. Debeer-Schmitt, and G. E. Granroth, Phys. Rev. B 91, 184513 (2015).

[10] M. Minola, G. Dellea, H. Gretarsson, Y. Y. Peng, Y. Lu, J. Porras, T. Loew, F. Yakhou, N. B. Brookes, Y. B. Huang, J. Pelliciari, T. Schmitt, G. Ghiringhelli, B. Keimer, L. Braicovich, and M. Le Tacon, Phys. Rev. Lett. 114, 217003 (2015).

[11] M. Guarise, B. D. Piazza, H. Berger, E. Giannini, T. Schmitt, H. M. Rønnow, G. A. Sawatzky, J. van den Brink, D. Altenfeld, I. Eremin, and M. Grioni, Nat. Commun. 5, 5760 (2014).

[12] M. P. M. Dean, A. J. A. James, A. C. Walters, V. Bisogni, I. Jarrige, M. Hücker, E. Giannini, M. Fujita, J. Pelliciari, Y. B. Huang, R. M. Konik, T. Schmitt, and J. P. Hill, Phys. Rev. B 90, 220506(R) (2014).

[13] M. P. M. Dean, A. J. A. James, R. S. Springell, X. Liu, C. Monney, K. J. Zhou, R. M. Konik, J. S. Wen, Z. J. Xu, G. D. Gu, V. N. Strocov, T. Schmitt, and J. P. Hill, Phys. Rev. Lett. 110, 147001 (2013).

[14] C. Monney, T. Schmitt, C. E. Matt, J. Mesot, V. N. Strocov, O. J. Lipscombe, S. M. Hayden, and J. Chang, Phys. Rev. B 93, 075103 (2016).

[15] H. Y. Huang, C. J. Jia, Z. Y. Chen, K. Wohlfeld, B. Moritz, T. P. Devereaux, W. B. Wu, J. Okamoto, W. S. Lee, M. Hashimoto, Y. He, Z. X. Shen, Y. Yoshida, H. Eisaki, C. Y. Mou, C. T. Chen, and D. J. Huang, Sci. Rep. 6, 19657 (2016).

[16] R. Zeyher and A. Greco, Phys. Rev. B 87, 224511 (2013).

[17] Q. Si, R. Yu, and E. Abrahams, Nature Reviews Materials 1, 16017 (2016).

[18] W. Chen and O. P. Sushkov, Phys. Rev. B 88, 184501 (2013).

[19] C. J. Jia, E. A. Nowadnick, K. Wohlfeld, Y. F. Kung, C.-C. Chen, S. Johnston, T. Tohyama, B. Moritz, and T. P. Devereaux, Nat. Commun. 5, 3314 (2014).

[20] F. Zhang, C. Gros, T. Rice, and H. Shiba, Supercon. Sci. Tech. 1, 36 (1988).
[21] P. A. Lee and N. Nagaosa, Phys. Rev. B 46, 5621 (1992).

[22] N. E. Bickers, Rev. Mod. Phys. 59, 845 (1987).

[23] Q. Yuan, Y. Chen, T. K. Lee, and C. S. Ting, Phys. Rev. B 69, 214523 (2004).

[24] Q. Yuan, T. K. Lee, and C. S. Ting, Phys. Rev. B 71, 134522 (2005).

[25] T. K. Lee, C.-M. Ho, and N. Nagaosa, Phys. Rev. Lett. 90, 067001 (2003).

[26] J.-X. Li, C.-Y. Mou, and T. K. Lee, Phys. Rev. B 62, 640 (2000).

[27] J.-X. Li, C.-Y. Mou, C.-D. Gong, and T. K. Lee, Phys. Rev. B 64, 104518 (2001).

[28] J.-X. Li and C.-D. Gong, Phys. Rev. B 66, 014506 (2002).

[29] J.-X. Li, J. Zhang, and J. Luo, Phys. Rev. B 68, 224503 (2003).

[30] M. U. Ubbens and P. A. Lee, Phys. Rev. B 46, 8434 (1992).

[31] J. Brinckmann and P. A. Lee, Phys. Rev. Lett. 82, 2915 (1999).

[32] T. K. Lee and C. T. Shih, Phys. Rev. B 55, 5983 (1997).

[33] D. S. Ellis, Y.-B. Huang, P. Olalde-Velasco, M. Dantz, J. Pelliciari, G. Drachuck, R. Ofer, G. Bazalitsky, J. Berger, T. Schmitt, and A. Keren, Phys. Rev. B 92, 104507 (2015).

[34] K. Ishii, M. Fujita, T. Sasaki, M. Minola, G. Dellea, C. Mazzoli, K. Kummer, G. Ghiringhelli, L. Braicovich, T. Tohyama, K. Tsutsumi, K. Sato, R. Kajimoto, K. Ikeuchi, K. Yamada, M. Yoshida, M. Kurooka, and J. Mizuki, Nat. Commun. 5, 3714 (2014).

[35] Y. Y. Peng, M. Hashimoto, M. M. Sala, A. Amorese, N. B. Brookes, G. Dellea, W.-S. Lee, M. Minola, T. Schmitt, Y. Yoshida, K.-J. Zhou, H. Eisaki, T. P. Devereaux, Z.-X. Shen, L. Braicovich, and G. Ghiringhelli, Phys. Rev. B 92, 064517 (2015).

[36] C. M. Varma, P. B. Littlewood, S. Schmitt-Rink, E. Abrahams, and A. E. Ruckenstein, Phys. Rev. Lett. 63, 1996 (1989).

[37] G. Kotliar and A. E. Ruckenstein, Phys. Rev. Lett. 57, 1362 (1986).

[38] C. Jia, K. Wohlfeld, Y. Wang, B. Moritz, and T. P. Devereaux, Phys. Rev. X 6, 021020 (2016).

[39] J. Brinckmann and P. A. Lee, Phys. Rev. B 65, 014502 (2001).

[40] Note that the AFM critical dopings for both electron-doped and hole-doped systems are roughly 0.15 . We do notice the overestimation of the AFM critical doping, especially in the hole-doped systems. This results from the lack of the competing order because the SC order is not taken into account when the AFM order is considered. In this way, the possible complexity of the phase coexistence can be avoided because, in this work, we mainly focus on the excitations in each given phase. See Ref. [39] for the phase diagram. For $\delta$ less than the critical doping, PM data are obtained by turning off the AFM channel in order to obtain the behavior in the PM phase in the given doping, as shown in Fig. 3 and Appendix A. 